\title{
Overview on the Future Electron-Ion Collider Projects
}

\author{
Emlyn W. Hughes \\ W.K. Kellogg Radiation Laboratory \\ California Institute of Technology \\ Pasadena, CA 91125
}

\begin{abstract}
One of the large new projects envisioned for the future US nuclear physics program is the construction of a high-energy electron ion collider. The motivation for such a machine would be primary directed at the study of the nucleon spin structure using polarized light ions and the study of low $\mathrm{x}$ gluon distributions in heavy nuclei. Two candidates coming from the two large DOE nuclear physics facilities, one at Brookhaven called eRHIC, and one at Jefferson Laboratory called ELIC are the primary candidates today.
\end{abstract}

\section{Introduction}

In November of 2003, the Secretary of Energy, Spencer Abraham, gave a speech to the US Congress outlining twenty-eight new large projects that represent the future science priorities for the DOE over the next 20 years [1]. Included on the list was an electron-ion collider (EIC). That was the good news. Unfortunately, the EIC only ranked \#23 and fell into the category of "long-term" projects. However, there is some substantial reality to this decision. Today we have two concepts of what an EIC might be. One is based upon an upgrade of an interaction region with a new electron accelerator in the existing polarized proton program at the Relativistic Heavy Ion Collider (RHIC) at Brookhaven. This upgrade is called eRHIC (electron collider at RHIC) [2]. The second involves the construction of a new polarized hadron accelerator that would be used to collide with the electron beam from CEBAF at Jefferson Laboratory. This project is called ELIC for Electron Light Ion Collider [3]. However, both Jefferson Lab and Brookhaven have two additional projects that appear on the DOE list ahead of the EIC. At CEBAF, a $12 \mathrm{GeV}$ upgrade of the beam energy is approved in spot \#7 and at Brookhaven, an upgrade of the RHIC heavy ion program is being proposed and sits at spot \#18. If one takes the DOE list seriously, then both the Brookhaven and Jefferson Lab "mid-term" projects will have to be done before an EIC is seriously considered. This places the EIC project well into the next decade.

In this paper, I will review briefly some of the global considerations for embarking on an EIC. The construction of a new collider in the US for nuclear physics would be based upon the interests of a broad community of physicists with a variety of topics that somehow unify into a single proposal. Now comes the most important sentence in the article. The strategy and opinions given in this paper are my own personal view of the projects and present-day situation and absolutely do not represent any community or group. So, let's start. 


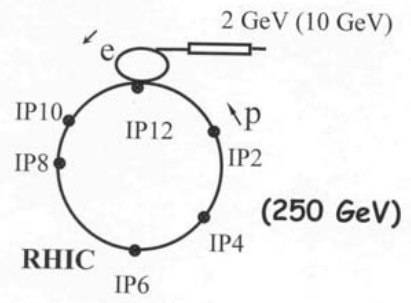

(a)

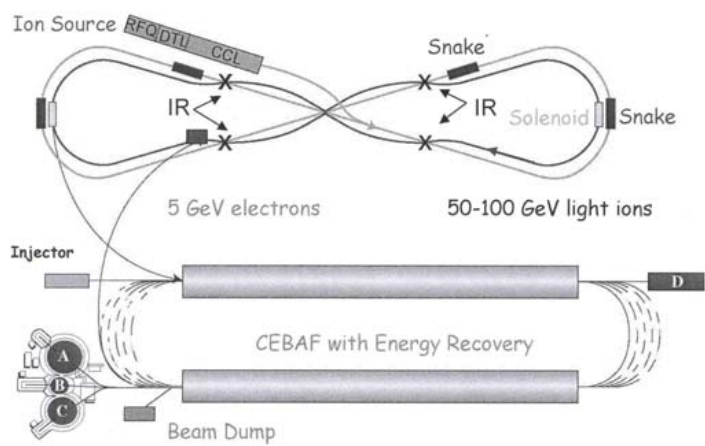

(b)

FIGURE 1. (a) Schematic of the eRHIC collider layout. (b) Schematic of the ELIC collider at Jefferson Lab.

\section{The Two EICs}

No matter where it is built, the EIC will cost one billion dollars.

The eRHIC proposal at present envisions a single interaction region in which a circular or possibly a linear electron accelerator is constructed to collide with the polarized proton or heavy ion beam produced at RHIC (Figure 1a). The project will require a large $4 \pi$ detector for studying the interactions. At a glance, the general idea of this proposal, to build a single ring and a single detector, should cost closer to $\$ 500$ million. The problem with the strawman eRHIC proposal is that (1) it assumes today's dollars and (2) it basically ignores the needs of the physics community interested in the study of low x structure functions of the proton and heavy ions. Although eRHIC is the highest energy electron-proton collider on the table today, its energy compared to HERA, for example, is limited (see Table 1). To continue the study of low x structure functions beyond HERA requires a dedicated forward-angle detector to access the low $\mathrm{x}$ region. A conventional $4 \pi$ detector will simply not fit into the same interaction region as a detector needed for low $\mathrm{x}$ structure function studies. The solution to this problem is to build two interaction regions at RHIC, one per program. But $2 \times \$ 500$ million is $\$ 1$ billion :)

To construct a new hadron machine at Jefferson Laboratory for ELIC (Figure 1b) simply costs $\$ 1$ billion; hadron machines are expensive. 
TABLE 1. EIC Parameters

\begin{tabular}{lcccc}
\hline Collider & $\begin{array}{c}\text { Luminosity } \\
\left(\mathbf{( 1 0}^{\mathbf{3 1}}\right)\end{array}$ & $\begin{array}{c}\text { Center of Mass } \\
\text { Energy } \mathbf{( G e V )}\end{array}$ & $\mathbf{Q}^{\mathbf{2}}$ & Bjorken x range \\
\hline HERA & 7 & 320 & 1 & $10^{-5}$ \\
& & & 5 & $10^{-4}$ \\
eRHIC & 50 & 100 & 1 & $10^{-4}$ \\
& & & 5 & $10^{-3}$ \\
ELIC & \multirow{2}{*}{$100-10,000$} & & 5 & $10^{-3}$ \\
& & & & $10^{-2}$ \\
\hline
\end{tabular}

The ELIC design has the nice feature of multiple interaction regions. Although this seems extravagant, multiple interaction regions give a wonderful boost to the nuclear community, similar to having multiple experimental halls. The freedom that multiple interaction regions provide should not be underestimated as a primary advantage of this idea.

However, ELIC operates at substantially lower center of mass energy. The machine will be able to perform detailed studies of the nucleon structure only in the mid $x$ range. An important consideration for the ELIC machine will be the study of energy upgrades. It is fine to start the collider at low energy and high luminosity, but without an upgrade to $6 \mathrm{GeV}$ electrons on, for example, $150 \mathrm{GeV}$ protons, the nucleon spin community will be limited. In particular, the kinematic coverage of ELIC may not extend out knowledge significantly compared to the fixed target programs that already have studied nucleon spin structure functions in the 1990s [4].

The subsequent four sections discuss how the two collider ideas stack up in addressing four mainline physics topics.

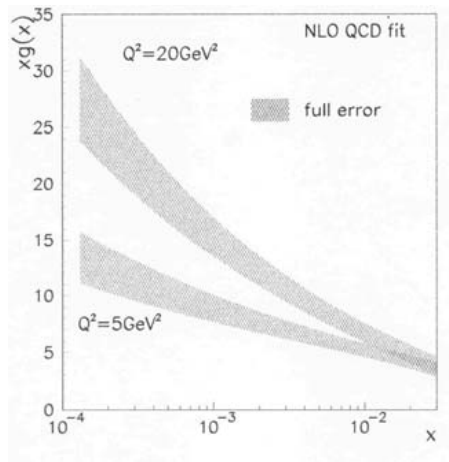

(a)

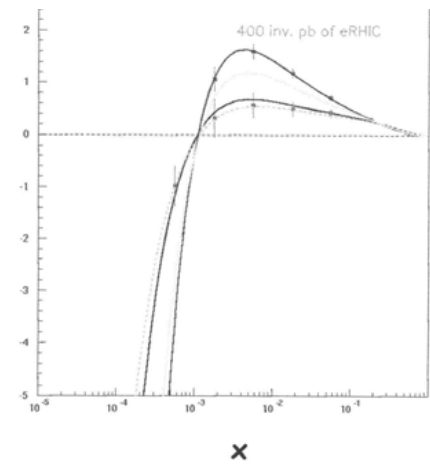

(b)

FIGURE 2. (a) Results on the unpolarized gluon distribution as measured by the H1 collaboration at HERA. (b) Simulation of the proton spin structure function results at low $\mathrm{x}$ from eRHIC for different polarized gluon distributions. 


\section{Polarized Gluon Distribution}

The proton spin structure can be decomposed into three pieces,

$$
\text { Proton spin }=1 / 2=1 / 2 \Delta q+\Delta G+L_{z}
$$

where $\Delta q$ corresponds to the quark contribution to the proton spin, $\Delta G$ is the gluon contribution and $\mathrm{L}_{\mathrm{z}}$ is the contribution from orbital angular momentum of the constituents. One of the greatest challenges today is to try to measure $\Delta \mathrm{G}$ for the first time. The expectation is that $\Delta \mathrm{G}$ should be quite large and positive, since the quark contribution only accounts for about 30\% of the total spin. RHIC Spin [5] and COMPASS [6] will make important first measurements of $\Delta G$ over the next five years, but the kinematic range of these measurements will be limited ( $\mathrm{x}>0.01)$, and theoretical uncertainties arising from the determination of structure functions in proton-proton scattering, for example, are likely to be large.

Measurements of $\mathrm{G}(\mathrm{x})$ from HERA represent the state-of-the art for determining the gluon distribution of the proton. Figure 2a presents results for $\mathrm{G}(\mathrm{x})$ coming from the $\mathrm{H} 1$ collaboration [7] evolved to an average $\mathrm{Q}^{2}$ of $5 \mathrm{GeV}^{2}$. The dream for the spin community is to produce exactly such a plot for $\Delta \mathrm{G}(\mathrm{x})$ coming from a polarized collider measurement. Figure $2 b$ shows a simulation of the precision on the proton spin structure function coming from the eRHIC program. The ability to make precision measurements of spin structure functions down to $\mathrm{x}$ of $10^{-3}$ is one of the main goals of the eRHIC program.

At present, with the limited center-of-mass energy, ELIC could not make a competitive measurement at low $\mathrm{x}$.

\section{Orbital Angular Momentum}

If the integral over the polarized gluon distribution gives a result that accounts for the remaining proton constituent contribution to its total spin, then the field of study will be quiet for awhile, and certainly any vestige of the "Proton Spin Puzzle" will disappear. However, if $\Delta \mathrm{G}$ is small or negative, there will still be a lively hunt to extract information on the quark or gluon orbital angular momentum contribution to the proton's spin.

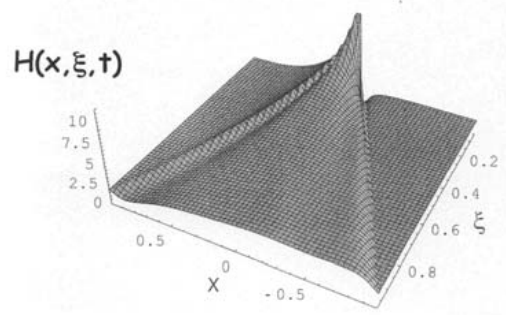

FIGURE 3. Sample GPD distribution as predicted from a theoretical model. 
Theoretically, the best hope today is that one may be able to find information on the quark orbital angular momentum contribution through deeply virtual Compton scattering [8] or, more generally, through the study of Generalized Parton Distributions (GPDs) [9]. Unfortunately, the measurements of these functions is very difficult and covers an enormous parameter space. Figure 3 presents a theorist vision of what one of these functions might look like. A primary goal of the Jefferson Laboratory $12 \mathrm{GeV}$ upgrade will be to make first extensive measurements of GPDs and try to evaluate whether this is a viable field of study.

Certainly, with the extended kinematic reach of an ELIC compared to the 12 GeV program, the GPD studies, if fruitful, could be continued with further precision at higher energies. For this idea to become a reality, a very high luminosity collider will be needed, and this makes ELIC a natural candidate, with it potential to reach $10^{35}$ $\mathrm{cm}^{-2}$ per second. This is to be contrasted to eRHIC which will not have a high luminosity compared to the $12 \mathrm{GeV}$ fixed-target program.

In fact, at present, it is probably reasonable to think of eRHIC as a collider to for measuring $\triangle \mathrm{G}$ and ELIC as a potential collider to study orbital angular momentum.

\section{Gluon Shadowing}

There has been a significant interest recently in the study of low x structure functions in the nucleon and nuclei and their implication for understanding unpolarized low x gluon distributions [10]. With limited space, I will just reference the analysis of HERA data [11] and the recent results from Brahms [12]. Results from these experiments indicate that the gluon distribution may be saturating at low $\mathrm{x}$ compared to the predictions of the DGLAP evolution equations [13-15]. It is intriguing that a heavy ion measurement can play a role in this study. Measurements made at the LHC will certainly be of enormous interest to continue probing at low $\mathrm{x}$.

If the study outlasts the heavy ion program at RHIC and the proton and heavy ion program at LHC, then eRHIC could make some important "clean" measurements of low x gluon distributions, especially in nuclei. Since ELIC is a low energy collider and only aimed at very light nuclei, this field of study is an eRHIC project.

But, will it still be interesting a decade from now, post LHC?

\section{Precision Electroweak}

I cannot resist, but end on a humorous note. First pointed out by Rolf Ent, ELIC has a luminosity that could, in principle, perform precision electroweak measurements via parity violation in polarized electron scattering from unpolarized protons. Figure 4 presents measurements of the electroweak mixing angle versus energy comparing what exists today and what an ELIC could do.

There is still a long list of difficulties that one would have to address even if the full luminosity ELIC collider worked flawlessly. Just to mention one, super precise polarimetry of the electron beam will be needed (also for an NLC, by the way!) Another problem is that many competitive precision parity-violation measurements will come earlier from the existing Jefferson Laboratory fixed target program plus experiments at the $12 \mathrm{GeV}$ upgrade. 


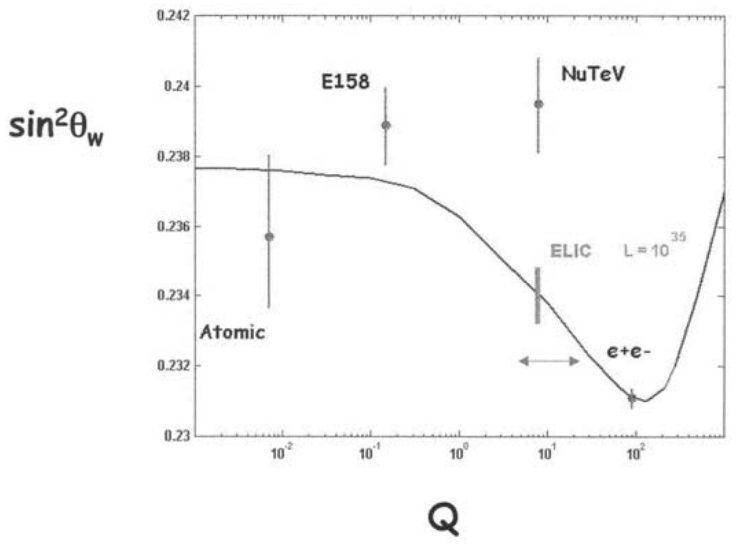

Figure 4. Measurements of the electroweak mixing parameter versus Q compared to a simulation from a possible measurement at the ELIC collider.

But, it is interesting and a testament to the new ideas possible at a very high luminosity collider. However, like the last section, after the LHC has finished its new particle searches, will this field of physics still be interesting?

\section{Summary}

The existence of an EIC in the US would certainly bring a significant community to collider physics for the study of strong interactions, taking up the flag where HERA will soon leave off. The biggest challenge faced by the proponents of an EIC today is to convince the world that this machine must be built in a reasonable timeframe.

\section{ACKNOWLEDGMENTS}

I would like to thank the conference organizers for the invitation to come to beautiful Marseille. A special thanks goes to both Zein-Eddine Meziani and Jacques Soffer for their enormous efforts. Also, I apologize that this collider discussion had nothing to do with high $\mathrm{x}$ ! After the $12 \mathrm{GeV}$ program at CEBAF and the high energy measurements from HERA, I do not believe that high $\mathrm{x}$ play a large role in the EIC business. I could certainly be wrong. HERA may still find leptoquarks at high $\mathrm{x}$, in which case all bets are off on the reason for building a US EIC. In that case, just shred this article. 


\section{REFERENCES}

1. Abraham, Spencer, talk given to US Congress, Nov. 10, 2003, Facilities for the Future of Science: A Twenty-year Oulook, http://www.er.doe.gov/Sub/Facilities_for_future/facilities_future.htm

2. R. Holt et al., The Electron-Ion Collider: A High Luminosity Probe of the Partonic Substructure of Nucleons and Nuclei, BNL-68933, Feb. 2002.

2. L. Merminga et al., ELIC: An Electron-Light Ion Collider Based at CEBAF, Proc. of EPAC, p. 203, Paris, France, 2002.

3. E.W. Hughes and R. Voss, Spin Structure Functions, Ann. Rev. Nucl. Part. Sci, 49, 303 (1999).

5. G. Bunce et al., Prospects for Spin Physics at RHIC, Ann. Rev. Nucl. Part. Sci, 50, 525 (2000).

6. G. Baum et al. (COMPASS Collaboration), Common Muon and Proton Apparatus for Structure and Spectroscopy, CERN/SPSLC 96-14, March 1996.

7. S. Aid et al. (H1 Collaboration), Nucl. Phys. B470, 3 (1996).

8. X. Ji, Int. J. Mod. Phys. A18, 1303 (2003).

9. X. Ji, Phys. Rev. Lett. 78, 610 (1997); Phys. Rev. D55, 7114 (1997).

10. J.-P. Blaizot \& F. Gelis, hep-ph/0405305, August 2004

11. L. Lindemann et al. (H1 and ZEUS Collaborations), Nucl. Phys. Proc. Suppl., 64, 179 (1998).

12. I. Arsene et al. (BRAHMS Collaboration), nucl-ex/0403005, March 2004.

13. G. Altarelli and G. Parisi, Nucl. Phys. B126, 298 (1977).

14. Yu, Dokshitzer, Sov. Phys. JETP 46, 641 (1977).

15. V.N. Gribov, L.N. Lipatov, Sov. J. Nucl. Phys. 15, 438 (1972); 15, 675 (1972). 